\title{
Esquema MIMO de Baixa Complexidade com Quatro Antenas Transmissoras e Taxa de Transmissão Unitária
}

\author{
Samuel T. Valduga, Dimas I. Alves, Renato Machado, Andrei P. Legg e Murilo B. Loiola
}

\begin{abstract}
Resumo-Neste artigo, propõe-se um esquema de transmissão MIMO (multiple-input multiple-output) de baixa complexidade com quatro antenas transmissoras e taxa de transmissão espacial unitária. Um pré-processador é projetado baseado na realimentação de fase provinda do receptor. $O$ pré-processamento permite que o esquema proposto alcance um grau de diversidade completo bem como um ganho de codificação. Realiza-se uma análise de SNR (signal-to-noise ratio) do sistema proposto a qual é utilizada para se encontrar a informação de fase ótima, no sentido de maximizar a SNR instantânea. Considera-se também uma análise do canal de realimentação quantizado supondo que o canal de comunicação é do tipo Rayleigh, plano e quase-estático. Realizam-se simulações do tipo Monte Carlo para o esquema proposto e para outros esquemas de comunicação MIMO com quatro antenas transmissoras e taxa de transmissão unitária. Resultados mostram que o esquema proposto alcança ordem de diversidade completa e supera outras boas propostas em termos de ganho de codificação e atraso de decodificação.
\end{abstract}

Palavras-Chave-Ganho de codificação, Sistemas MIMO, Realimentação de fase, STBC.

Abstract-In this paper we propose a low-complexity rateone multiple-input multiple-output (MIMO) scheme with four transmit antennas. A preprocessor design based on phase feedback information enables the proposed scheme to achieve full diversity order as well as a coding advantage. A signal-tonoise ratio (SNR) analysis is performed and it is used to find the optimal (non-quantized) phases in the sense of maximizing the instantaneous SNR. A quantized feedback analysis of the proposed scheme for quasi-static flat Rayleigh fading channels is also considered. Monte Carlo simulations are performed for different closed-loop space-time block codes with four transmit antennas and unitary transmission rate. Results illustrate that the proposed scheme achieves full diversity order and outperforms other good schemes in terms of coding gain and decoding delay.

Keywords-Coding gain, MIMO systems, phase feedback, STBC.

\section{INTRODUÇÃO}

Para quem atua na área de codificação de canal, já é bem conhecido que sistemas com múltiplas antenas podem aumentar a capacidade do sistema e reduzir os efeitos do

Samuel T. Valduga, Dimas I. Alves, Renato Machado e Andrei P. Legg, Grupo de Pesquisa em Comunicações e Processamento de Sinais, Departamento de Eletrônica e Computação, Universidade Federal de Santa Maria, Santa Maria, RS, 97105-900, Brasil. Email: samucatv@gmail.com, dirion@lacesm.ufsm.br, renatomachado@ufsm.br, andrei.legg@gmail.com. Murilo B. Loiola, Universidade Federal do ABC, Santo André, SP, 09210-170, Brasil. E-mail: murilo.loiola@ufabc.edu.br. Esse trabalho foi financiado em partes pelo CNPq e pela FAPERGS. desvanecimento com a exploração inteligente da dimensão espacial. Em [1], Alamouti propôs o primeiro código de bloco espaço temporal ortogonal (OSTBC), o qual explora a diversidade espacial no transmissor. A taxa máxima de um STBC complexo com $M_{T}=2^{n} M$ antenas transmissoras, em que $M$ é um número ímpar e $n$, um número inteiro, é $\frac{n+1}{M_{T}}$ [2]. Quando $M_{T}=2$, o código de Alamouti é o código ótimo, além de ser o único OSTBC complexo de taxa unitária.

OSTBCs não requerem o conhecimento do estado do canal (CSI, channel state information) no transmissor para proporcionar a máximo ganho de diversidade. Enquanto a restrição de ortogonalidade é necessária para se obter o ganho de diversidade máximo, é visto em [4] que quando essa restrição é relaxada maiores taxas de transmissão podem ser obtidas com o custo de perda de algum grau na ordem de diversidade.

Múltiplas antenas transmissoras podem melhorar significativamente o desempenho dos sistemas de comunicação sem fio se houver um canal de retorno que informe ao transmissor a CSI do sistema [6], [7]. Ao longo dos últimos anos, vários trabalhos vêm investigando o uso inteligente do canal de realimentação em sistemas com múltiplas antenas. Alguns destes trabalhos são rapidamente descritos a seguir.

\section{A. Trabalhos Relacionados}

Em [8], Machado and Uchôa-Filho propuseram um esquema de seleção híbrida antena/código no transmissor que seleciona, a partir de uma lista de códigos espaço temporais de bloco, o melhor código e um certo subconjunto de antenas transmissoras para realizar a transmissão dos símbolos naquele quadro de transmissão. O código selecionado é baseado no critério da mínima probabilidade de erro instantânea, ou seja, o código escolhido para aquele quadro de transmissão deve garantir que a probabilidade de erro instantânea seja a mínima possível. Esta idéia foi refinada posteriormente em [9].

Esquemas de comunicação que exploram o uso de múltiplas antenas transmissoras e canal de realimentação quantizado são candidatos para compor o padrão LTE [5]. Vários artigos propondo técnicas de comunicação que consideram o uso de canal de realimentação podem ser facilmente encontrados [10]-[13].

Em [12], uma extensão dos OSTBC (EO-STBCs) para sistemas MIMO com quatro antenas transmissoras foi proposta. Nesse trabalho, a informação de fase é enviada do receptor ao transmissor e baseado nesta informação, as fases de 
certos símbolos são rotacionadas afim de maximizar a razão sinal-ruído (SNR) instantânea. A taxa de transmissão deste código é unitária, uma vez que dois símbolos de informação são enviados a cada dois períodos de símbolo. Como resultado, o EO-STBC apresentado em [12] superou o desempenho de outros STBCs de taxa unitária que já haviam sido propostos.

Em [13],Choi et al. propuseram uma interessante estratégia de comunicação que também explora o uso do canal de realimentação quantizado. Nesta proposta um pré-processador é usado para combinar dois códigos de Alamouti no sentido de se maximizar a norma de Frobenius (termo presente na SNR). Eles mostraram que a diversidade completa é alcançada, além de um ganho de codificação. Neste artigo, este esquema é denominado Esquema Baseado no Código de Alamouti (ACBS, Alamouti code based scheme).

\section{B. Objetivos e Contribuições}

Neste artigo, propõe-se um esquema com quatro antenas transmissoras, taxa de transmissão unitária e canal de realimentação de fase. Projeta-se um pré-processador que é atualizado pela realimentação de fase a cada novo quadro de transmissão. O pré-processamento é realizado com o intuito de maximizar a SNR instantânea. O esquema proposto baseia-se na combinação das propostas apresentadas em [12] e [13].

Uma análise da SNR instantânea para o esquema proposto é apresentada. Com esta análise, pode-se encontrar a configuração ótima para o pré-processador. Cabe ressaltar que o esquema proposto possui um receptor de baixa complexidade, cuja detecção é baseada em processamento linear simples. Uma avaliação de desempenho do esquema proposto considerando um canal de retorno quantizado foi realizado também. Resultados de simulações revelam que o esquema proposto alcança a diversidade máxima e supera o desempenho de outros bons STBCs (com mesma configuração) que foram previamente apresentados na literatura.

\section{Organização do Trabalho e Algumas Notações}

O restante deste trabalho está organizado da seguinte forma. A Seção II apresenta o modelo do sistema. A Seção III apresenta o esquema proposto neste trabalho, bem como a análise de sua SNR. Na Seção IV, apresentam-se os resultados de simulação. Finalmente, a Seção V conclui o artigo.

Ao longo deste artigo, letras normais representam quantidades escalares, letras minúsculas em negrito indicam vetores, letras maiúsculas em negrito indicam matrizes e os sobrescritos ' $\mathrm{T}$ ' $\mathrm{e}$ ' $*$ ' representam a operação transposta $\mathrm{e}$ conjugado complexo, respectivamente.

\section{Modelo do Sistema}

Considere um sistema MIMO com $M_{T}=4$ antenas transmissoras e $M_{R}$ antenas receptoras. O canal é considerado plano, quase estático e com desvanecimento Rayleigh. O modelo de transmissão é semelhante ao descrito em [13]. Com algumas modificações chegamos em

$$
\mathbf{Y}=\mathbf{X H I}_{S N R}+\mathbf{N}
$$

em que $\mathbf{Y}$ é a matriz $\tau \times M_{R}$ dos sinais recebidos, $\mathbf{X}$ é a matriz $\tau \times M_{T}$ de sinais transmitidos com energia média unitária. $\mathrm{A} \mathcal{C} \mathcal{N}(0, \mathbf{R})$ representa a p.d.f. (probability density function) conjunta de um vetor de variável aleatória normal, circularmente simétrico com matriz de covariância $\mathbf{R}$. $\mathbf{N}$ é a matriz $\tau \times M_{R}, \mathcal{C N}\left(\mathbf{0}, \mathbf{I}_{\tau N_{R}}\right)$ representando a p.d.f. conjunta de amostras do ruído Gaussiano Branco i.i.d. (independent and identically distributed) com variância média unitária. $\mathbf{H}$ é a matriz canal MIMO $M_{T} \times M_{R}$, caracterizada pela p.d.f. $\mathcal{C N}\left(\mathbf{0}, \mathbf{I}_{M_{T} M_{R}}\right)$, e $\mathbf{I}_{S N R}$ é a matriz diagonal $M_{R} \times M_{R}$, em que o $i$-ésimo elemento diagonal desta matriz é dado por $\sqrt{\rho}$, com $\rho=E_{s} / N_{0}$, em que $E_{s}$ é a energia média do símbolo, e $N_{0}$ é a variância do ruído.

Assume-se que os bits de informação são mapeados em uma constelação de símbolos em banda-base de energia média unitária, como a PSK ou QAM, dando origem à $Q$ símbolos $\left\{s_{q}\right\}, q=1, \ldots, Q$, os quais serão transmitidos durante $\tau$ períodos de símbolo. Neste artigo, consideram-se apenas esquemas com taxa de transmissão espacial unitária $(R=$ $Q / \tau=1$ ). Assumem-se também que os coeficientes do canal são perfeitamente conhecidos no receptor e que existe um canal de realimentação através do qual $b$ bits podem ser enviados para o transmissor.

\section{ESQUEMA MIMO DE BAIXA COMPLEXIDADE COM Quatro ANTENAS TRANSMissoras E TAXA DE TRANSMISSÃO UNITÁRIA}

Nesta seção, apresenta-se o esquema proposto, onde o préprocessador linear é apresentado primeiro e, posteriormente, a proposta do receptor de baixa complexidade para o caso $M_{R}=1$ e $M_{R}=2$.

\section{A. O Transmissor}

$\mathrm{O}$ esquema proposto tem quatro antenas transmissoras, através das quais um novo símbolo de informação, $s$, é transmitido em cada novo período. O símbolo $s$ é préprocessado pelo vetor $\mathbf{p}$, resultando em vetor complexo $\mathbf{x}$ :

$$
\begin{aligned}
\mathbf{x} & =\mathbf{p} s \\
& =\left[\mathbf{v}_{\varphi}\left(\left[\begin{array}{cc}
\cos (\theta) / \sqrt{2} & 0 \\
0 & \sin (\theta) / \sqrt{2}
\end{array}\right] \otimes \mathbf{I}_{2}\right)\right] s \\
& =\frac{1}{\sqrt{2}}\left[\begin{array}{c}
\cos (\theta) e^{j \varphi_{0}} \\
\cos (\theta) e^{j \varphi_{1}} \\
\sin (\theta) e^{j \varphi_{0}} \\
\sin (\theta) e^{j \varphi_{2}}
\end{array}\right]^{\mathrm{T}} s
\end{aligned}
$$

em que $\otimes$ é o produto de Kronecker, $\mathbf{I}_{2}$ é a matriz identidade 2 por 2 , e

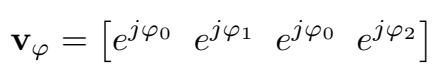

O fator $\frac{1}{\sqrt{2}}$ é usado para normalizar a potência de transmissão. Com o intuito de simplificar a análise, assumi-se que $\varphi_{0}=0$. Essa simplificação é válida, uma vez que $\varphi_{0}$ é apenas um ângulo de referência.

Antes de cada transmissão ocorrer, o receptor envia $b$ bits para o transmissor. Esta informação é utilizada pelo préprocessador para a escolha das fases apropriadas $\left(\varphi_{1}, \varphi_{2} \mathrm{e}\right.$ $\theta$ ) no sentido de se maximizar SNR instantânea. 


\section{B. Receptor}

Nesta seção, apresenta-se o receptor proposto para $M_{R}=1$ e $M_{R}=2$, respectivamente. A análise da SNR é apresentada e utilizada para se obter a informação de fase que maximiza a SNR instantânea.

1) Receptor linear para uma antena receptora: Das Equações (1) and (2), o sinal recebido y para $M_{R}=1$ torna-se

$$
y=\operatorname{sh}_{e q} \sqrt{\rho}+\eta
$$

sendo

$$
\begin{aligned}
h_{e q} & =\mathbf{p h}, \\
& =\frac{\left(h_{1,1}+h_{2,1} e^{j \varphi_{1}}\right) \cos (\theta)+\left(h_{3,1}+h_{4,1} e^{j \varphi_{2}}\right) \sin (\theta)}{\sqrt{2}}
\end{aligned}
$$

em que $\mathbf{h}=\left[\begin{array}{lll}h_{1,1} & \ldots & h_{4,1}\end{array}\right]^{T}, \eta$ é o ruído aditivo Gaussiano branco, e $h_{i, j}$ denota o ganho do canal entre a $i$-ésima antena transmissora e a $j$-ésima antena receptora.

Considere o sinal recebido em (3). O seguinte processamento linear produz as entradas desejadas para o detector de máxima verossimilhança (ML):

$$
\begin{aligned}
\tilde{s}= & y h_{e q}^{*} \\
= & s\left[\left(\left|h_{1,1}\right|^{2}+\left|h_{2,1}\right|^{2}+\beta_{c}\right) \cos ^{2}(\theta)\right. \\
& +\left(\left|h_{3,1}\right|^{2}+\left|h_{4,1}\right|^{2}+\beta_{s}\right) \sin ^{2}(\theta) \\
& \left.\quad+\beta_{c s} \cos (\theta) \sin (\theta)\right] / 2+h_{e q}^{*} \eta
\end{aligned}
$$

com

$$
\begin{aligned}
\beta_{c} & =2 \Re\left\{h_{1,1} h_{2,1}^{*} e^{-j \varphi_{1}}\right\} \\
\beta_{s} & =2 \Re\left\{h_{3,1} h_{4,1}^{*} e^{-j \varphi_{2}}\right\} \\
\beta_{c s} & =2 \Re\left\{\left(h_{1,1}+h_{2,1} e^{j \varphi_{1}}\right)\left(h_{3,1}+h_{4,1} e^{j \varphi_{2}}\right)^{*}\right\}
\end{aligned}
$$

em que $|\cdot|^{2}$ denota o módulo ao quadrado de um número complexo e $\Re\{\cdot\}$, a parte real de um número complexo. Como é possível se observar acima, a detecção pode ser realizada com baixa complexidade.

a) Análise da SNR: Para o sinal apresentado em (4), a SNR instantânea é dada por

$$
\mathrm{SNR}=\frac{g_{c} \cos ^{2}(\theta)+g_{s} \sin ^{2}(\theta)+\beta_{c s} \cos (\theta) \sin (\theta)}{2}
$$

em que

$$
\begin{aligned}
& g_{c}=\left|h_{1,1}\right|^{2}+\left|h_{2,1}\right|^{2}+\beta_{c} \\
& g_{s}=\left|h_{3,1}\right|^{2}+\left|h_{4,1}\right|^{2}+\beta_{s}
\end{aligned}
$$

Agora, pode-se determinar as fases, $\varphi_{1}, \varphi_{2}$ e $\theta$, ótimas a partir da derivada de (8), resultando em uma máxima SNR instantânea e ordem de diversidade $4\left(M_{T}=4\right.$ e $\left.M_{R}=1\right)$. Contudo, cabe salientar que a máxima diversidade também pode ser alcançada com a realimentação quantizada (conforme é descrito posteriormente).

$\varphi_{1}, \varphi_{2}$, e $\theta$, são as variáveis consideradas neste processo de otimização. De (8), observa-se que existe uma dependência entre estas variáveis, tornando a maximização da SNR instantânea um problema um pouco mais difícil de se resolver. Assim, optou-se por resolver este problema em dois passos, conforme é descrito a seguir.
Primeiro, derivam-se $\beta_{c}$ e $\beta_{s}$ em função de $\varphi_{1}$ e $\varphi_{2}$, respectivamente, e igualam-se ambos a zero. Assim, pode-se facilmente verificar que estes termos são maximizados quando

$$
\varphi_{1}=\xi_{1,1}-\xi_{2,1} \quad \text { e } \quad \varphi_{2}=\xi_{3,1}-\xi_{4,1}
$$

em que $h_{i, j}=\alpha_{i, j} \exp \left\{j \xi_{i, j}\right\}$.

Segundo, deriva-se (8) em função de $\theta$, seguindo o procedimento similar ao adotado em [13]. A primeira e segunda derivada são dadas por

$$
S N R^{\prime}=2 \kappa(\cos (\theta) \sin (\theta))+\beta_{c s}\left(\cos ^{2}(\theta)-\sin ^{2}(\theta)\right)
$$

$$
S N R^{\prime \prime}=2 \kappa\left(\cos ^{2}(\theta)-\sin ^{2}(\theta)\right)-4 \beta_{c s}(\cos (\theta) \sin (\theta))
$$

Resolvendo (11) e (12) e considerando as condições de contorno $S N R^{\prime}=0$ e $S N R^{\prime \prime}<0$, obtém-se o theta ótimo ${ }^{1}$

$$
\theta_{o p t}=\arctan \left(\frac{\kappa+\sqrt{\kappa^{2}+2 \beta_{c s}^{2}}}{\beta_{c s}}\right)
$$

com

$$
\kappa=g_{s}-g_{c}
$$

Foi decidido omitir o termo $\rho$ em (11) e (12), uma vez que a omissão deste não altera o resultado final.

2) Receptor linear para duas antenas receptoras: Das Equações (1) and (2), o sinal recebido y para $M_{R}=2$ antenas receptoras pode ser reescrito como

$$
\mathbf{y}=s \mathbf{h}_{e q} \mathbf{I}_{S N R}+\mathbf{n}
$$

em que $\mathbf{h}_{e q}=\mathbf{p H}=\left[\begin{array}{ll}h_{e q 1} & h_{e q 2}\end{array}\right]^{\mathrm{T}}, \mathbf{y}=\left[\begin{array}{ll}y_{1} & y_{2}\end{array}\right]$, e

$$
\mathbf{H}=\left[\begin{array}{llll}
h_{1,1} & h_{2,1} & h_{3,1} & h_{4,1} \\
h_{1,2} & h_{2,2} & h_{3,2} & h_{4,2}
\end{array}\right]^{\mathrm{T}}
$$

Considere o sinal recebido em (15). O seguinte processamento linear produz as entradas desejadas para o detector ML:

$$
\begin{aligned}
\tilde{s}= & y_{1} h_{e q 1}^{*}+y_{2} h_{e q 2}^{*} \\
= & s\left[\left(\left|h_{1,1}\right|^{2}+\left|h_{2,1}\right|^{2}+\left|h_{1,2}\right|^{2}+\left|h_{2,2}\right|^{2}+\beta_{c}\right) \cos (\theta)^{2}\right. \\
& +\left(\left|h_{3,1}\right|^{2}+\left|h_{4,1}\right|^{2}+\left|h_{3,2}\right|^{2}+\left|h_{4,2}\right|^{2}+\beta_{s}\right) \sin (\theta)^{2} \\
& \left.+\beta_{c s} \cos (\theta) \sin (\theta)\right] / 2+h_{e q 1}^{*} \eta_{1}+h_{e q 2}^{*} \eta_{2}
\end{aligned}
$$

$\operatorname{com} \beta_{c}=\beta_{c 1}+\beta_{c 2}, \quad \beta_{s}=\beta_{s 1}+\beta_{s 2}, \quad \beta_{c s}=\beta_{c s 1}+\beta_{c s 2}, \mathrm{e}$

$$
\begin{aligned}
& \beta_{c 1}=2 \Re\left\{h_{1,1} h_{2,1}^{*} e^{-j \varphi_{1}}\right\} \\
& \beta_{c 2}=2 \Re\left\{h_{1,2} h_{2,2}^{*} e^{-j \varphi_{1}}\right\} \\
& \beta_{s 1}=2 \Re\left\{h_{3,1} h_{4,1}^{*} e^{-j \varphi_{2}}\right\} \\
& \beta_{s 2}=2 \Re\left\{h_{3,2} h_{4,2}^{*} e^{-j \varphi_{2}}\right\} \\
& \beta_{c s 1}=2 \Re\left\{\left(h_{1,1}+h_{2,1} e^{j \varphi_{1}}\right)\left(h_{3,1}+h_{4,1} e^{j \varphi_{2}}\right)^{*}\right\} \\
& \beta_{c s 2}=2 \Re\left\{\left(h_{1,2}+h_{2,2} e^{j \varphi_{1}}\right)\left(h_{3,2}+h_{4,2} e^{j \varphi_{2}}\right)^{*}\right\}
\end{aligned}
$$

\footnotetext{
${ }^{1}$ Ótimo no sentido de se maximizar a SNR instantânea.
} 
a) Análise da SNR: Para o sinal obtido em (17), a SNR instantânea é dada por

$$
\mathrm{SNR}=\frac{g_{c} \cos ^{2}(\theta)+g_{s} \sin ^{2}(\theta)+\beta_{c s} \cos (\theta) \sin (\theta)}{2}
$$

em que

$$
\begin{aligned}
& g_{c}=\left|h_{1,1}\right|^{2}+\left|h_{2,1}\right|^{2}+\left|h_{1,2}\right|^{2}+\left|h_{2,2}\right|^{2}+\beta_{c} \\
& g_{s}=\left|h_{3,1}\right|^{2}+\left|h_{4,1}\right|^{2}+\left|h_{3,2}\right|^{2}+\left|h_{4,2}\right|^{2}+\beta_{s}
\end{aligned}
$$

Podem-se determinar novamente o $\varphi_{1}, \varphi_{2}$, e $\theta$ ótimos a partir da derivada de (24), resultando em uma máxima SNR instantânea e ordem de diversidade $8\left(M_{T}=4\right.$ e $\left.M_{R}=2\right)$.

Seguindo os mesmos procedimentos de cálculo considerados para o caso $M_{R}=1$, obtém-se a solução em dois passos. Primeiro, derivamos $\beta_{c}$ e $\beta_{s}$ em função de $\varphi_{1}$ e $\varphi_{2}$, respectivamente, e igualam-se ambos a zero. Assim, esses termos são maximizados quando

$$
\begin{aligned}
& \varphi_{1}=\arctan \left(\frac{\tan \left(\xi_{1,1}-\xi_{2,1}\right)}{\Gamma_{1}+\Delta_{1}} \Gamma_{1}+\frac{\tan \left(\xi_{1,2}-\xi_{2,2}\right)}{\Gamma_{1}+\Delta_{1}} \Delta_{1}\right) \\
& \varphi_{2}=\arctan \left(\frac{\tan \left(\xi_{3,1}-\xi_{4,1}\right)}{\Gamma_{2}+\Delta_{2}} \Gamma_{2}+\frac{\tan \left(\xi_{3,2}-\xi_{4,2}\right)}{\Gamma_{2}+\Delta_{2}} \Delta_{2}\right)
\end{aligned}
$$

em que

$$
\begin{aligned}
& \Gamma_{1}=\frac{\cos \left(\xi_{1,1}-\xi_{2,1}\right)}{\cos \left(\xi_{1,2}-\xi_{2,2}\right)}, \Delta_{1}=\frac{\alpha_{1,2} \alpha_{2,2}}{\alpha_{1,1} \alpha_{2,1}} \\
& \Gamma_{2}=\frac{\cos \left(\xi_{3,1}-\xi_{4,1}\right)}{\cos \left(\xi_{3,2}-\xi_{4,2}\right)}, \Delta_{2}=\frac{\alpha_{3,2} \alpha_{4,2}}{\alpha_{3,1} \alpha_{4,1}}
\end{aligned}
$$

Segundo, deriva-se (24) em função de $\theta$. A primeira e segunda derivadas são dadas por

$$
S N R^{\prime}=2 \kappa(\cos (\theta) \sin (\theta))+\beta_{c s}\left(\cos ^{2}(\theta)-\sin ^{2}(\theta)\right)
$$

$\mathrm{e}$

$$
S N R^{\prime \prime}=2 \kappa\left(\cos ^{2}(\theta)-\sin ^{2}(\theta)\right)-4 \beta_{c s}(\cos (\theta) \sin (\theta))
$$

Resolvendo (27) e (28) e considerando as condições de contorno $S N R^{\prime}=0$ e $S N R^{\prime \prime}<0$, obtém-se o theta ótimo

$$
\theta_{o p t}=\arctan \left(\frac{\kappa+\sqrt{\kappa^{2}+2 \beta_{c s}^{2}}}{\beta_{c s}}\right)
$$

com

$$
\kappa=g_{s}-g_{c}
$$

A extensão desta análise para mais de duas antenas receptoras é semelhante.

\section{Realimentação Quantizada}

Nesta seção, apresenta-se como implementar o esquema proposto considerando um canal de realimentação quantizado. Assume-se duas quantizações de fase: $\varphi_{1 q}$ e $\varphi_{2 q} \in[0, \pi]$ e $\theta_{q} \in[-\pi / 2, \pi / 2]$. O receptor processa e compara $2^{b-2}$ valores de SNR instantânea e envia $b$ bits de informação para o transmissor. É importante ressaltar que o receptor precisa enviar as informações sobre as 3 fases, assim o transmissor recebe no mínimo 3 bits de realimentação para o pré-processamento da informação (detalhes podem ser vistos na Tabela I).
TABELA I

REAlimentaÇÃo QuAntizada: Critério PARA A SEleÇÃo de Fase.

\begin{tabular}{c|c|c|c}
\hline Numero de bits de realimentação & $\beta_{c}$ & $\beta_{s}$ & $\varphi_{1 q} / \varphi_{2 q}$ \\
\hline \multirow{4}{*}{$b=2$ bits } & $>0$ & $>0$ & $0 / 0$ \\
\cline { 2 - 4 } & $>0$ & $<0$ & $0 / \pi$ \\
\cline { 2 - 4 } & $<0$ & $>0$ & $\pi / 0$ \\
\cline { 2 - 4 } & $<0$ & $<0$ & $\pi / \pi$ \\
\hline+ & $\beta_{c s}$ & $\kappa$ & $\theta_{q}$ \\
\hline \multirow{2}{*}{1 bit } & $>0$ & $\sharp$ & $\frac{\pi}{4}$ \\
\cline { 2 - 4 } & $<0$ & $\sharp$ & $\frac{-\pi}{4}$ \\
\hline ou + & $\beta_{c s}$ & $\kappa$ & $\theta_{q}$ \\
\hline \multirow{2}{*}{2 bits } & $>0$ & $>0$ & $\frac{3 \pi}{8}$ \\
\cline { 2 - 4 } & $>0$ & $<0$ & $\frac{\pi}{8}$ \\
\cline { 2 - 4 } & $<0$ & $>0$ & $\frac{-\pi}{8}$ \\
\cline { 2 - 4 } & $<0$ & $<0$ & $\frac{-3 \pi}{8}$ \\
\hline
\end{tabular}

A Tabela I mostra como melhorar o desempenho do esquema proposto para $b=3$ e 4 bits de realimentação. Nesta tabela, define-se o critério de escolha da informação da fase que será utilizada na transmissão. As fases $\varphi_{1}$ e $\varphi_{2}$ são usadas para garantir que os termos $\beta_{c}$ e $\beta_{s}$ sejam números positivos. A terceira fase, $\theta$ é usada para maximizar a soma dos termos que compõem a SNR instantânea. O símbolo $\sharp$ especifica "estado indiferente". Desta forma, $\beta_{c}, \beta_{s}$ e $\theta$ convergem para a solução ideal (não-quantizado) conforme a quantização é aumentada.

\section{Resultados de SimulaçÃo}

Nesta seção, os resultados de simulação ilustram o desempenho do esquema proposto. No intuito de avaliar a diversidade e o ganho de codificação, comparam-se os resultados de simulação do esquema proposto com os resultados dos esquemas EO-STBC [12] e ACBS [13]. O desempenho foi comparado em termos de taxa de erro de bit (BER) versus $\rho$ em canais com desvanecimento Rayleigh, planos e quase estáticos. Nas Fig. 1 e 2, apresentam-se os resultados para $M_{T}=4$ antenas transmissoras e $M_{R}=1$ antena receptora. Na Fig. 3, apresentam-se os resultados para $M_{T}=4$ antenas transmissoras e $M_{R}=1, M_{R}=2$ antenas receptoras. Nas simulações, assumem-se que o símbolos são mapeados em uma constelação 4-QAM e o critério de parada é a ocorrência de 300 erros de símbolos recebidos a cada ponto de SNR média. Os esquemas apresentam taxa de transmissão unitária, ou seja, para constelações $M$-QAM, os esquemas possuem taxa de transmissão $R=\frac{Q}{\tau} \log _{2}(M)=\log _{2}(M)$. Como curva de referência, considera-se a curva de BER para o cenário sem diversidade (SISO) em todas as figuras. Para todas as simulações assume-se que os bits enviados pelo canal de realimentação não contém erros.

$\mathrm{Na}$ Fig. 1, apresentam-se o desempenho de BER do esquema proposto e dos esquemas EO-STBC e ACBS. Podese observar que o esquema proposto alcança ordem máxima de diversidade, igual a 4 (a mesma alcançada pelos outros esquemas) e tem um ganho de codificação em torno de $2.4 \mathrm{~dB}$ sobre os esquemas EO-STBC e ACBS. Na Fig. 2, apresenta-se a BER alcançada pelo esquema proposto para $b=3$ bits de realimentação (Veja Tabela I). O esquema proposto tem um ganho de desempenho de aproximadamente $1.6 \mathrm{~dB}$ sobre os esquemas EO-STBC e ACBS. 


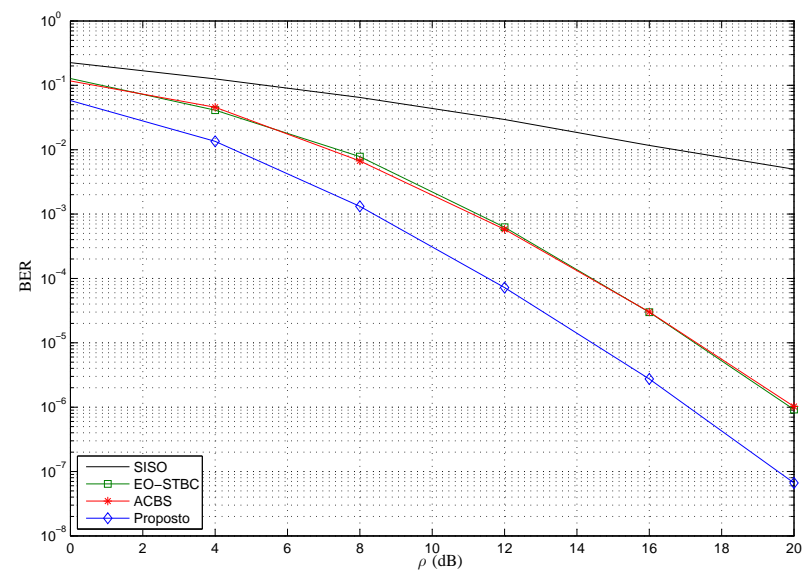

Fig. 1. Realimentação de fase ideal (sem quantização), $M_{T}=4, M_{R}=1$.

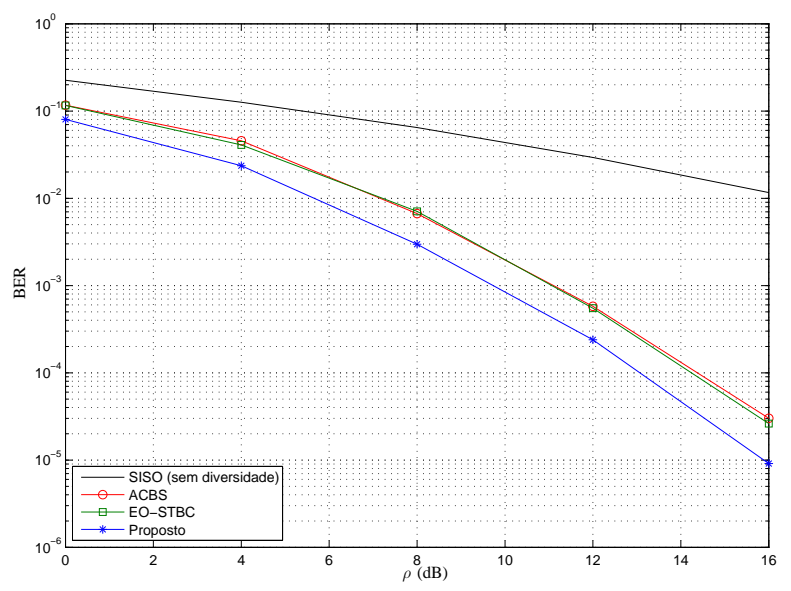

Fig. 2. Desempenho da BER. $M_{T}=4, M_{R}=1$ and $\mathrm{b}=3$.

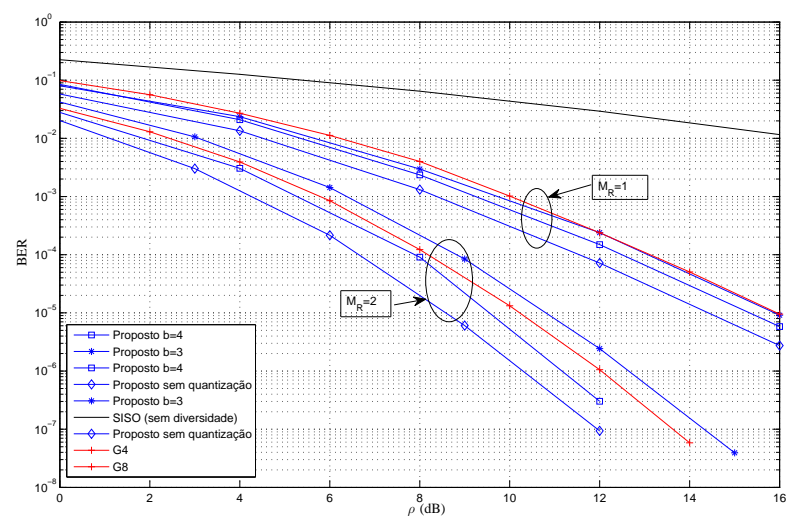

Fig. 3. Desempenho de BER do esquema proposto, $M_{R}=1$ and $M_{R}=2$.

A Fig. 3 mostra as curvas de BER com ordem de diversidade igual a 4 e 8 para o esquema proposto com $M_{R}=1 \mathrm{e}$ $M_{R}=2$, respectivamente. O esquema tem uma melhora de desempenho de acordo com o aumento do número de bits de realimentação. $\mathrm{O}$ resultado obtido para o caso ideal (sem quantização) é apresentada nesta figura também. Foram apresentadas as curvas dos OSTBC G4 e G8, propostos por Tarokh [3], com diversidade 4 e 8, respectivamente. As curvas de BER para os códigos G4 e G8 são aplicados em sistemas com quatro antenas transmissores e uma antena receptora e quatro antenas transmissoras e duas antenas receptoras, respectivamente. Esses OSTBC apresentam taxa de transmissão igual a 1/2. Logo, as curvas de desempenho desses OSTBC são utilizadas apenas como curvas de referência de ordem máxima de diversidade.

\section{Conclus Ão e CONSIDERaÇões Finais}

Neste artigo, um esquema MIMO de baixa complexidade com quatro antenas transmissoras, canal de realimentação quantizado e taxa de transmissão unitária foi proposto. A ideia principal voltada para esta proposta foi explorar os benefícios do esquema [12] combinado com o esquema [13] em uma nova estratégia de diversidade de transmissão. Uma análise de SNR foi feita e posteriormente utilizada para se encontrar as fases ótimas, como intuito de se maximizar a SNR instantânea. Além disso, uma avaliação do desempenho do sistema considerando um canal de realimentação quantizado foi realizada. Seu desempenho foi avaliado através de simulações do tipo Monte Carlo. Finalmente, observou-se que o esquema proposto alcança máxima diversidade e supera outros bons esquemas de transmissão em termos de ganho de codificação.

\section{AGRADECIMENTOS}

Os autores agradecem ao CNPq e à FAPERGS pelo apoio financeiro concedido para a realização deste trabalho.

\section{REFERÊNCIAS}

[1] S. M. Alamouti, "A simple transmit diversity technique for wireless communications," IEEE J. Select. Areas Commun., vol. 16, pp. 14511458, Oct. 1998.

[2] X-B. Liang, "Orthogonal designs with maximal rates," IEEE Trans. on Inf. Theory, vol. 49, pp. 2468-2503, Oct. 2003.

[3] V. Tarokh, H. Jafarkhani, and A. R. Calderbank, "Space-Time block codes from orthogonal designs," IEEE Trans. Inform. Theory, vol. 45, no. 5, pp. 1456-1467, Jul. 1999.

[4] M. Uysal and C. N. Georghiades, "Non-Orthogonal space-time block codes for 3Tx antennas," IEEE Electronics Lett., vol. 38, no. 25, pp. 1689-1691, Dec. 2002.

[5] http://www.3gpp.org/Future-Radio-in-3GPP-300-attend

[6] G. Caire, G. Taricco, and E. Biglieri, "Optimum power control over fading channels," IEEE Trans. Inf. Theory, vol. 45, no. 5, pp. 14681489, Jul. 1998.

[7] Z. Chen, J. Yuan, and B. Vucetic, "Analysis of transmit antenna selection/maximal-ratio combining in Rayleigh fading channels," IEEE Trans. Vehic. Tecnology, vol. 54, no. 4, pp. 1312-1321, Jul. 2005.

[8] R. Machado and B. F. Uchôa-Filho, "Space-time block coding with hybrid transmit antenna/code selection," in Proc. IEEE Int. Conf. on Commun. (ICC'04), 2004, pp. 819-822.

[9] J. Akhtar and D. Gesbert, "Extending orthogonal block codes with partial feedback," IEEE Trans. Wireless Commun., vol. 3, no. 6, pp. 1959-1962, Nov. 2004.

[10] R. W. Heath Jr. and A. Paulraj, "A simple scheme for transmit diversity using partial channel feedback," Proc. 32nd Asilomar Conf. on Signals, Systems, and Computers, Nov. 1998, pp. 2441-2444.

[11] C. R. Murthy and D. Rao, "Quantization methods for equal gain transmission with finite rate feedback," IEEE Trans. Signal Processing, vol. 55, no. 1, pp. 233-245, Jan. 2007.

[12] N. M. Eltayeb, S. Lambotharan, and J. A. Chambers, "A phase feedback based extended space-time block code for enhancement of diversity," in Proc. IEEE VTC'07, April 2007, pp. 2296-2299.

[13] I. Choi, Jong-Kyu Kim, H. Lee, and I. Lee, "Alamouti-codes based four-antenna transmission schemes with phase feedback," IEEE Communications Letters, vol. 13, no. 10, pp. 749-751, Oct. 2009. 